\title{
Retrosternal goitre: Anaesthetic implications and management
}

\author{
Nitin Choudhary ${ }^{1, *}$, Abhijit Kumar ${ }^{2}$, Sonia Wadhawan ${ }^{3}$, Poonam Bhadoria $^{4}$, Vishnu Panwar ${ }^{5}$ \\ ${ }^{1}$ Senior Resident, ${ }^{2} \mathrm{PG}$ Student, ${ }^{3,4}$ Director Professor, ${ }^{5}$ Specialist, Dept. of Anaesthesiology, Maulana Azad Medical College, New \\ Delhi, India
}

*Corresponding Author:

Email: drnitinchoudhary@yahoo.in

Received: $13^{\text {th }}$ May, 2017

Accepted: $29^{\text {th }}$ December, 2017

\begin{abstract}
Large retrosternal goitre is a challenge to the anaesthesiologist and the surgeon. We describe the successful anaesthetic management of a 44 year old male patient with extensive retrosternal goitre with severe tracheal compression, operated for total thyroidectomy under general anaesthesia by combined cervical approach and sternotomy. A multidisciplinary team approach with surgical colleagues allowed successful management of the patient.
\end{abstract}

Keywords: Retrosternal goitre, Thyroidectomy, Sternotomy, Awake fibreoptic intubation.

\section{Introduction}

Huge goitres have a dramatic appearance but often present fewer problems than smaller retrosternal goitres. ${ }^{1}$ Retrosternal goitre (RSG) may be classified depending on the location: grade 1 , above the aortic arch; grade 2, between the aortic arch and pericardium; and grade 3, below the right atrium. ${ }^{2}$ Retrosternal goitres usually cause compression of mediastinal structures and may also cause serious complications like cerebral hypoperfusion and axillosubclavian vein thrombosis. ${ }^{3}$ A surgical approach is usually via a cervical incision but, depending upon intra-thoracic extension, it may necessitate manubriotomy, sternotomy or thoracotomy (3.1, 6.6 and 4\%, respectively) $)^{2}$ which exposes the patient to the risk of pneumothorax and haemorrhage.

Anaesthesia for thyroid surgery requires an anaesthetist who is experienced in the recognition, assessment, and management of a potentially difficult, shared airway, in a patient who may also have significant co-morbidity. We describe the anaesthetic management of a massive retrosternal goitre with severe intrathoracic tracheal narrowing scheduled for total thyroidectomy.

\section{Case Report}

A 44 year old male $(170 \mathrm{~cm}, 90 \mathrm{~kg}, \mathrm{BMI}=31.1$ $\mathrm{kg} / \mathrm{m}^{2}$ ) having diffuse colloid goitre with retrosternal extension was posted for total thyroidectomy. Patient complained of swelling in the neck since 6-7 years not associated with symptoms or signs of mediastinal compression, hypothyroidism or hyperthyroidism. Patient was a known smoker (15-20 pack years) and known diabetic since 6 months on oral hypoglycaemic agents.

General physical examination was unremarkable. Local examination revealed a firm neck swelling measuring $12 \times 15 \mathrm{~cm}$ with dilated superficial veins. No bruit was heard and lower border of swelling was not palpable. Neck movements were restricted. Patient had adequate mouth opening with MMP III. On Indirect laryngoscopy, bilateral vocal cords were mobile. Other systemic examination did not show any abnormality.

Blood count, electrocardiogram, blood sugar levels, renal/liver function test, thyroid function test, serum calcium, coagulation profile and arterial blood gas were within normal limits. Chest radiograph showed well defined lobulated soft tissue opacity with base towards mediastinum in right paratracheal location suggesting mediastinal origin. X-ray soft tissue neck lateral view revealed increased soft tissue density in posterior tracheal space at $\mathrm{C}_{4}-\mathrm{C}_{6}$ level causing widening and deviation of airway towards left side. CECT (Contrast enhanced computed tomography) of neck reported diffuse enlargement of thyroid gland with right lobe measuring $5.8 \times 6.8 \times 15.9 \mathrm{~cm}$ with retrosternal extension measuring $7.5 \times 7.4 \times 5.5 \mathrm{~cm}$ and left lobe measuring $5.5 \times 5.6 \times 11.6 \mathrm{~cm}$ without retrosternal extension (Fig. 1). In addition, gland produced mass effect in the form of deformation and attenuation of tracheal lumen and posterior displacement of great vessels of neck. No mass infiltration into the airway was noted. 


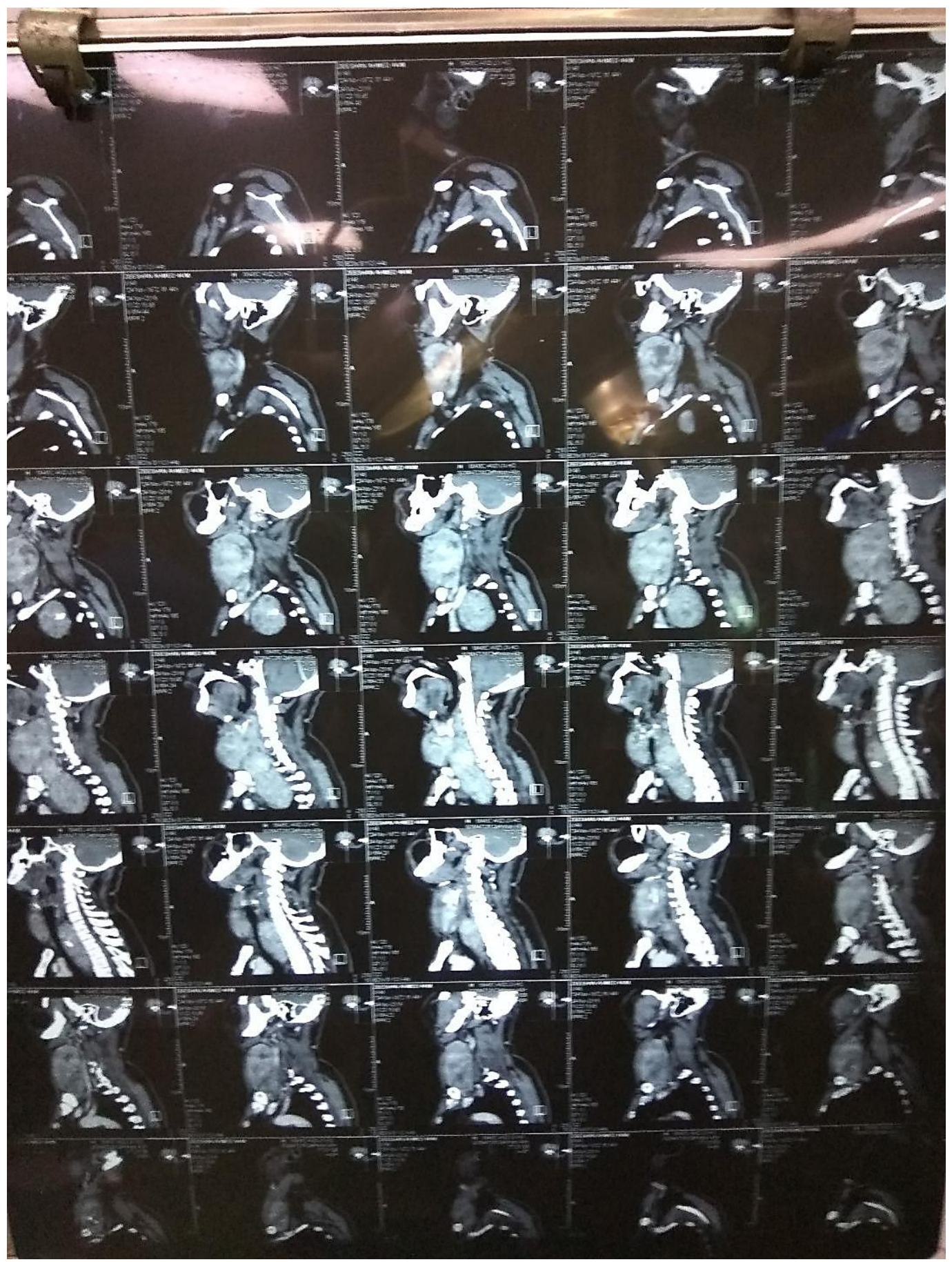

Fig. 1

Preoperatively, patient was advised to stop smoking. Patient's blood sugar were well controlled on OHA. Patient was advised to omit the morning dose of OHA on the day of surgery. Chest physiotherapy and incentive spirometry were started. Patient was started on bronchodilator therapy. He was taken up for surgery under ASA II. Patient was explained about the need for elective mechanical ventilation post surgery in view of the nature of the surgery, the risks of difficult airway and consequences of hypoxia and mediastinal mass syndrome following induction of anaesthesia. Standard
NPO orders were followed. Adequate blood and blood products were ensured on the day of surgery.

Multiple perioperative challenges in the form of difficult airway, possibility of acute cardiorespiratory decompensation (anterior mediatinal syndrome), significant blood loss, postoperative pain management and rehabilitation required a comprehensive contingency plan preoperatively involving the anaesthesiologist, intensivist, ENT surgeon, CTVS surgeon. Morning blood sugar level on the day of surgery was $98 \mathrm{mg} / \mathrm{dl}$. In the preoperative room, patient was prepared for awake nasal fibreoptic intubation. 
Injection glycopyrrolate $200 \mu \mathrm{g}$ IM was given. Patient was nebulised with $5 \mathrm{ml}$ of $4 \%$ lignocaine and oral cavity sprayed with $10 \%$ lignocaine. After checking nasal patency, right nostril was prepared using $2 \%$ xylometazoline nasal drops. Patient shifted inside the operation theatre, standard ASA monitors (NIBP, ECG, pulse oximeter) attached. Large bore16G cannula was secured over dorsum of right hand and normal saline was started. Oxygen was administered via nasal prongs at $4 \mathrm{~L} / \mathrm{min}$. Patient was given sitting position and under all aseptic conditions, thoracic epidural was placed at $\mathrm{T}_{7}-\mathrm{T}_{8}$ level. Left radial artery was cannulated and PICC line was placed in right antecubital fossa. The ENT surgeon was standby in the operating room to initiate rigid bronchoscopy and jet ventilation in the event of failed intubation. Difficult airway cart was kept ready. Awake flexible bronchoscopy showed significant compression in mid-trachea region and patient was intubated with $8.0 \mathrm{~mm}$ cuffed reinforced endotracheal tube which was placed beyond the region of tracheal compression. Subsequently patient was induced with injection propofol $80 \mathrm{mg}$ IV. Injection fentanyl $2 \mu \mathrm{g} / \mathrm{kg}$ IV was given. Patient was maintained on $\mathrm{O}_{2}+\mathrm{N}_{2} \mathrm{O}+$ Isoflurane (MAC $\leq 1$ ). Adequate muscle relaxation was achieved with injection vecuronium. Patient was positioned for surgery with neck extension for adequate surgical exposure. Patient was catheterised for perioperative fluid management. Intraoperative controlled hypotension was provided with injection nitroglycerine infusion and analgesia maintained with epidural bupivacaine top-ups and intravenous morphine. Perioperatively blood sugars were maintained between $120-180 \mathrm{mg} / \mathrm{dl}$ using vellore regimen. CTVS surgeon performed sternotomy during which patient was off ventilation to avoid any lung injury and was ventilated intermittently after informing the surgeon. Subsequently normal ventilation was resumed. Once the tumour was excised, leak was detected on ventilation and the cuff was further inflated (excluding tracheomalacia). The $650 \mathrm{gm}$ tumour was successfully removed via cervical and retrosternal approach with 1.2 litres blood loss. Blood loss was adequately replaced with fluids. Patient was shifted to ICU for overnight elective mechanical ventilation. On $1^{\text {st }}$ postoperative day patient was weaned off the ventilator and extubated under fibreoptic guidance to assess the functioning of vocal cords and reconfirm absence of tracheomalacia. There was mild edema of vocal cords and patient was started on adrenaline nebulisation. Postoperative pain was managed with NSAIDs and epidural morphine $30 \mu \mathrm{g} / \mathrm{kg}$ based on verbal rating scale $(\mathrm{VRS} \geq 3)$. Incentive spirometry and chest physiotherapy were started. Patient was mobilised on $2^{\text {nd }}$ postoperative day and was shifted to ward. Patient was discharged after a week of uneventful stay.

\section{Discussion}

The concerns with huge retrosternal goitres include mediastinal mass syndrome, difficult intubation, massive blood loss, prolonged surgical time, sternotomy concerns and post thyroidectomy complications.

Our patient did not present with any symptom of mediastinal compression however the presence of massive retrosternal goitre should raise the anaesthetic concerns pertaining to mediastinal mass syndrome. Thorough preoperative evaluation to rule out signs and symptoms of mediastinal mass syndrome is important aspect of successful management in such patients. In these cases CECT is very helpful in evaluation and successful management of the airway. The incidence of difficult intubation in patients with goitres is $2-12 \% .^{4,5}$ Awake techniques to secure the airway includes awake fibreoptic intubation(AFOI), awake tracheostomy and, very rarely, awake laryngoscopy and awake rigid bronchoscopy(RB). Awake tracheostomy is technically difficult to perform in the presence of an enlarged and vascular thyroid gland, but it can be lifesaving. In our patient keeping in mind the possible complications, awake fibreoptic intubation was the safest way to secure the airway before giving general anaesthesia for surgery. Topical anaesthesia of the airway ensured patient's cooperation for smooth conduct of awake fibreoptic intubation. Airway blocks were not feasible in our patient. ENT surgeon was prepared for surgical airway in case we failed to intubate. The availability of rigid bronchoscopy with jet ventilation would offer rescue in the event of loss of airway control. ${ }^{6}$ Each case needs to have a tailored "plan $\mathrm{ABC}$ " after weighing the risks and benefits. Cook et al. showed that there is no consensus on the best 'plan A' to secure the airway in patients with goitre, even among international airway experts. $^{7}$

In view of the anticipated blood loss and the risk of cardiovascular decompensation, invasive haemodynamic monitoring was done by placing an arterial catheter and PICC (peripherally inserted cenral catheter) line. Patient with mediastinal mass can collapse at any stage perioperatively, so vigilant monitoring is mandatory in these patients. Availability of adequate blood and blood products should always be ensured before taking up the patient for surgery. Sternotomy is a very painful procedure which requires adequate analgesia, deeper planes of anaesthesia and good muscle relaxation to spread the sternum. During sternotomy there are chances of vascular and lung injury. Anaesthetist should intermittently stop the ventilation at the time of sternotomy and resume ventilation as and when required till the time the procedure is ongoing.

In our patient, there was gross handling of the trachea intraoperatively. In addition, there was significant blood loss and there was a possibility of hypocalcaemia (following parathyroid gland excision). 
In lieu of the above reasons, we planned to electively ventilate the patient for 24 hours to allow airway oedema to settle and help in smooth extubation of the patient. The thoracic epidural provided adequate pain relief perioperatively and patient could resume deep breathing exercises and incentive spirometry following extubation; decreasing the chances of postoperative pulmonary complications.

In conclusion, patients with goitre are common and, in extreme cases, may present unique set of challenges to the anaesthetist. Careful consideration of the disease process (and its secondary effects), the patient's detailed history and examination, and relevant investigation are essential. A comprehensive preoperative planning and optimisation with a close working relationship among multidisciplinary medical teams were prerequisite for successful perioperative management and uneventful recovery of this patient. A structured in-house clinical protocol should be developed and deployed in order to ensure safe management of such patients in the future.

\section{References}

1. D. Nakra, G.D. Puri. Anaesthetic management of retrosternal goitre. J Anaesth Clin Pharmacol. 2005;21(3):309-311.

2. Huins CT, Georgalas C, Mehrzad H. A new classification system for retrosternal goitre based on a systematic review of its complications and management. Int J Surg 2008;6:71-76.

3. Santos GH, Ghalili K. Axillosubclavian vein thrombosis produced by retrosternal thyroid. Chest. 1990;98(5):12813.

4. Randolph GW, Shin JJ, Grillo HC, et al. The surgical management of goiter: Part II. Surgical treatment and results. Laryngoscope. 2011;121:68-76.

5. Chaves A, Carvalho Sand Botelho M. Difficult endotracheal intubation in thyroid surgery: A retrospective study. Internet J Anesthesiol. 2009;22(1).

6. Choa YK, Liu YH, Hsieh MJ, Wu YC, Liu HP, Wang CI, et al. Controlling difficult airway by rigid bronchoscopean old but effective method. Interact Cardiovasc Thorac Surg. 2005;4:175-9.

7. Cook TM, Morgan PJ and Hersch PE. Equal and opposite expert opinion. Airway obstruction caused by a retrosternal thyroid mass: Management and prospective international expert opinion. Anaesthesia. 2011;66:826836.

How to cite this article: Choudhary N, Kumar A, Wadhawan S, Bhadoria P, Panwar V. Retrosternal goitre: Anaesthetic implications and management. Indian J Clin Anaesth. 2018;5(3):453-456. 